\title{
PAPER
}

\section{Sensitivity and specificity of the new international diagnostic criteria for migraine with aura}

\author{
M K Eriksen, L L Thomsen, J Olesen
}

J Neurol Neurosurg Psychiatry 2005;76:212-217. doi: 10.1136/jnnp.2004.037853

\begin{abstract}
See end of article for authors' affiliations

.....................

Correspondence to: Dr M K Eriksen, Danish Headache Center, University of Copenhagen, Department of Neurology, Glostrup Hospital, Nordre Ringvej 57, DK-2600 Glostrup, Denmark; kirchmann@dadlnet.dk
\end{abstract}

Received 27 January 2004 In revised form 20 April 2004 Accepted 11 May 2004
Objectives: Since 1998, migraine with aura (MA) has been diagnosed according to the operational diagnostic criteria of the International Headache Society (ICHD-1). Here we present the data underlying the new criteria for MA in the ICHD-2 classification.

Methods: Sensitivity of the new criteria was tested in patients with MA and specificity in patients with reversible non-aura visual disturbances. The diagnoses in both groups of patients were made in a validated semistructured physician-conducted interview. We tested five sets of criteria for sensitivity and specificity comparing with the diagnosis according to the ICHD-1 in 200 patients and the selected set of criteria in 274 additional patients.

Results: Four sets of criteria had sensitivity/specificity of $46 \% / 100 \%, 71 \% / 100 \%, 62 \% / 95 \%$, and $99 \%$ / $76 \%$. Sensitivity of the selected set of criteria was $84 \%(95 \% \mathrm{Cl} 79 \%$ to $90 \%)$ and specificity $97 \%(95 \% \mathrm{Cl}$ $95 \%$ to $99 \%$ ). According to these criteria at least two of the following should be fulfilled: homonymous visual or unilateral sensory symptoms; at least one aura symptom develops gradually over $\geqslant 5$ minutes and/or different symptoms occur in succession over $\geqslant 5$ minutes; each symptom lasts $\geqslant 5$ and $\leqslant 60$ minutes. In the additional sample sensitivity of the selected criteria was $90 \% 195 \% \mathrm{Cl} 86 \%$ to $94 \%$ ) and specificity $96 \%$ (95\% Cl $91 \%$ to $100 \%$ ).

Conclusions: The diagnostic criteria for MA selected for ICHD-2 had high sensitivity and specificity. The ICHD-2 criteria are more operational and probably delineate a more homogeneous sample of patients than the ICHD-1. The ICHD-2 for MA is intended equally for research and clinical practice and can be used at different levels of specialisation.
M igraine with aura (MA) has been diagnosed according to the operational diagnostic criteria of the International Headache Society since 1988 (International Classification of Headache Disorders (ICHD)-1) (table 1). The diagnosis of MA relies exclusively on the description of symptoms because there are no diagnostic biological markers available to confirm the diagnosis. The principles of the ICHD- 1 for all primary and secondary headaches have been recognised in clinical practice ${ }^{2-6}$ and have been adopted by the World Health Organization (WHO) (ICD-10 NA). ${ }^{7}$ The ICHD- 1 has been universally accepted and translated into more than 20 languages and there is no other competing headache classification.

The ICHD-1 criteria for MA were mostly based on expert opinion due to the scarcity of empirical studies. ${ }^{8}$ During the work on the second edition of the ICHD (ICHD- 2$)^{9}$ it appeared that the ICHD-1 criteria for MA were difficult to understand and did not describe the aura in detail. Therefore, the reliability of the diagnosis of aura could very likely be improved. Furthermore, the ICHD-1 for MA had a major error as patients could be diagnosed as having MA according to the criteria without fulfilling the criterion for presence of any typical symptom of aura.

For several years our group has collected data on patients with MA diagnosed according to the ICHD-1 criteria for genetic studies. Validated, semistructured telephone interviews were conducted by a trained physician and generated detailed data on the migraine aura. Part of these data were used in a preliminary search for diagnostic criteria for MA with an optimal combination of sensitivity and specificity, and the selected criteria were included in the ICHD-2 (table 2). In the present study we present the sensitivity, specificity, and likelihood ratios for the new criteria for MA compared with the old criteria using a larger sample of patients than previously. We calculated all relevant parameters in two large independent samples-that is, the sample used in the preliminary search for reliable MA criteria for the ICHD-2 plus an additional sample used for validation of the criteria.

\section{PATIENTS AND METHODS}

\section{Phenotype delineation}

Patients were diagnosed as having MA if they fulfilled the ICHD-1 for MA and their aura was characterised by fully reversible visual symptoms, sensory symptoms, or dysphasic speech disturbances (that is, impaired production of language, impaired comprehension of language). Patients with hemiplegic aura were excluded since hemiplegic migraine is different from MA and therefore diagnosed according to separate diagnostic criteria in the ICHD-2. ${ }^{9-11}$

\section{Data collection}

The patients were recruited by a computerised search of the National Patient Register and screening of 27000 case records from headache clinics and practising neurologists. Only patients with MA from families with a least one affected sib pair or patients with hemiplegic migraine were recruited. ${ }^{10-12}$ The 1831 recruited patients were sent a letter with information about the project before they were contacted by telephone. Of these 1831 patients, 85 patients were non-contactable and 381 patients did not participate. ${ }^{12}$ The remaining 1365 patients (called probands) took part in a screening telephone interview (fig 1): 980 probands were diagnosed as having MA of whom 189 had a family history of an MA sib pair. ${ }^{12}{ }^{13}$ Selected relatives and probands from

Abbreviations: ICHD, International Classification of Headache Disorders; MA, migraine with aura 
Table 1 Diagnostic criteria for migraine with aura according to the International Headache Society classification, 1988 (ICHD-1)'

(A) At least two attacks fulfilling (B)

(B) At least three of the following four characteristics:

One or more fully reversible aura symptoms indicating cerebral cortical-and/or brain stem dysfunction

At least one aura symptom develops gradually over more than four minutes or two or more symptoms occur in succession

No aura symptom lasts more than 60 minutes. If more than one aura symptom is present, accepted duration is proportionally increased

Headache follows aura with a free interval of less than 60 minutes. (It may also begin before or simultaneously with the aura)

(C) At least one of the following:

History and physical and neurological examination not suggestive of one of the disorders listed in groups 5-11

History and/or physical and/or neurological examination suggest such disorder, but it is ruled out by appropriate investigations

Such disorder is present, but migraine attacks do not occur for the first time in close temporal relation to the disorder

these families were contacted for an extensive validated semistructured telephone interview ${ }^{14}$ with a trained physician. ${ }^{13}$ Of the 736 relatives selected 25 were non-contactable and 68 did not participate. The remaining 643 relatives took part in an interview. ${ }^{13}$ In total, 105 probands and 257 of their relatives were diagnosed as having MA according to the ICHD-1 in an extensive interview and participated in the present study. ${ }^{13}$ Furthermore, 112 patients with other reversible visual disturbances related to headache were included in the study. Their visual disturbances were not judged to be visual migraine aura and they did not fulfil the ICHD-1 for MA. These patients were identified during the initial screening telephone interviews of the probands (57 patients) and during the extensive telephone interviews of the relatives ( 55 patients). Thus our study population comprised 474 patients: 362 patients with MA and 112 patients with non-aura reversible visual disturbances.

The project was approved by the Danish ethical committees. Further details about the sample, non-participation, and comparison with a representative population based sample have been reported elsewhere. ${ }^{12} 13$

\section{Data processing and statistical analysis}

The 474 participants were divided into two subsamples: the training sample of 200 patients comprised 141 patients with MA plus 59 patients with non-aura visual disturbances (the 200 participants enrolled when the present study was initiated) and the validation sample of 274 patients comprised 221 patients with MA plus 53 patients with non-aura visual disturbances (the participants enrolled afterwards). The training sample was used for testing several sets of selected diagnostic criteria for MA all comprising three aura characteristics selected a priori in general agreement by the Classification Committee of the International Headache Society:
(1) Homonymous visual symptoms or unilateral sensory symptoms

(2) At least one aura symptom develops gradually over $\geqslant 5$ minutes and/or different symptoms occur in succession over $\geqslant 5$ minutes

(3) Each symptom lasts $\geqslant 5$ minutes and $\leqslant 60$ minutes

We aimed at identifying the diagnostic criteria for MA comprising the combination of the three aura characteristics with the highest sensitivity and specificity when compared with the diagnosis according to the ICHD-1 for MA. Subsequently, the accepted criteria were validated on the validation sample.

For the sake of simplicity and because of pressure of time the diagnoses made according to the selected diagnostic criteria in the training sample were based exclusively on the visual symptoms of the patients. However, the diagnoses made according to the accepted criteria in the validation sample were based on the visual, sensory, and aphasic aura symptoms. Statistical analyses were performed using SPSS Base System 11.5 for Windows XP Professional.

\section{RESULTS}

\section{Characteristics of patients with MA}

The 362 patients with MA comprised 99 men and 263 women (M:F ratio 1:2.7; mean age 46 (SD 16) years, range 12-90). At least in some attacks $99 \%$ (358/362) of patients had visual aura; 54\% (196/362) had sensory aura; and 32\% (116/362) had aphasic aura. Most patients had a combination of aura symptoms, since $28 \%$ (102/362) had co-occurring visual and sensory aura; $25 \%$ (91/362) had visual, sensory, and aphasic aura; $6 \%$ (23/362) had visual and aphasic aura; $1 \%$ (4/362) had other aura combinations; and 39\% (142/362) had visual aura exclusively. The characteristics of the symptoms of aura are shown in table 3. Overall, $88 \%(319 / 362)$ of the patients

Table 2 Diagnostic criteria for migraine with typical aura according to the International Classification of Headache Disorders Second Edition (ICHD-2) ${ }^{9}$

(A) At least two attacks fulfilling criteria (B)-(D)

(B) Aura consisting of at least one of the following but no motor weakness:

Fully reversible visual symptoms including positive features (that is, flickering lights, spots, lines) and/or negative features (scotoma)

Fully reversible sensory symptoms including positive features (that is, pins and needles) and/or negative features (numbness)

Fully reversible dysphasic speech disturbance

(C) At least two of the following:

Homonymous visual symptoms and/or unilateral sensory symptoms

At least one aura symptom develops gradually over $\geqslant 5$ minutes and/or different symptoms occur in succession over $\geqslant 5$ minutes

Each symptom lasts $\geqslant 5$ minutes and $\leqslant 60$ minutes

(D) This criterion determines the subdiagnosis of migraine with typical aura:

1.2.1 Typical aura with migraine headache: Headache fulfilling criteria B-D for 1.1 Migraine without aura begins during the aura or follows aura within

60 minutes, or

1.2.2 Typical aura with non-migraine headache: Headache that does not fulfil criteria B-D for 1.1 Migraine without aura begins during the aura or follows aura within 60 minutes, or

1.2.3 Typical aura without headache: Headache does not occur during the aura nor follow aura within 60 minutes

(E) Not attributed to another disorder 


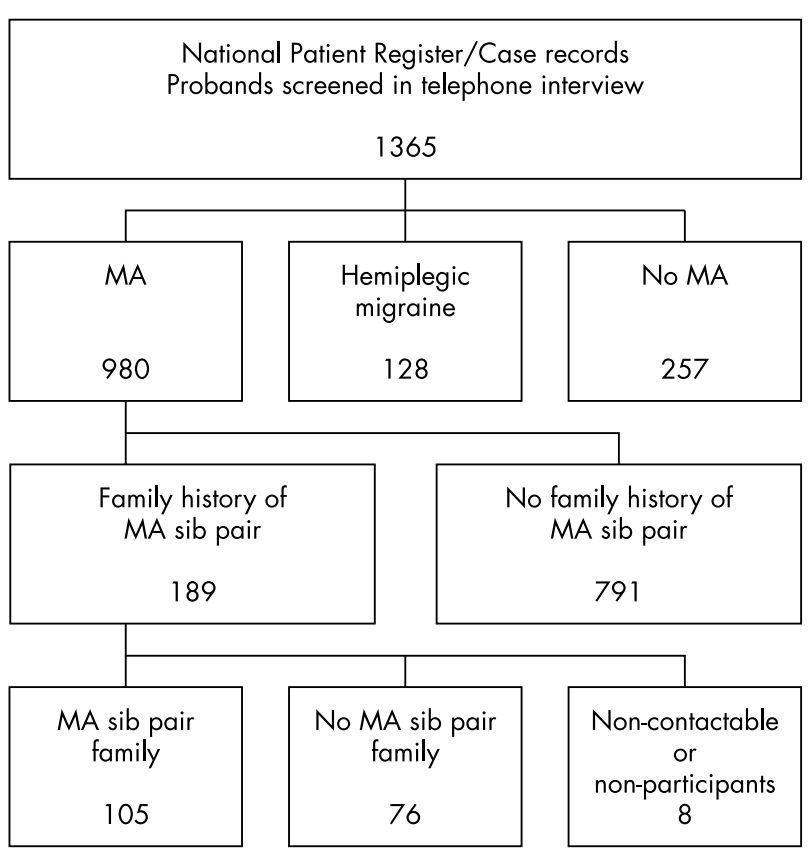

Figure 1 Ascertainment of probands with non-hemiplegic migraine with aura (MA). In total, 105 families with at least one MA sib pair were identified. The patients with MA included in the present study were recruited from these families.

with MA fulfilled the ICHD- 1 criterion of a gradual development of the aura, 83\% (300/362) fulfilled the criterion of aura duration, and 94\% (340/362) had a headache following the aura with a free interval of less than one hour. When more than one aura symptom was observed, they occurred in succession in 96\% (149/155) and simultaneously in $4 \%(6 / 155)$ of patients (65 missing values or patients uncertain). The headache related to aura began after the onset of the aura in $82 \%(278 / 341)$, simultaneously with the aura in $11 \%(37 / 341)$ and before the onset of the aura in $8 \%$ $(26 / 341)$ of patients. A total of 21 patients had aura without headache exclusively.
Characteristics of patients with non-aura reversible visual disturbances

The 112 patients with non-aura reversible visual disturbances comprised 32 men and 80 women (M:F ratio 1:2.5; mean age 41 (14) years, range 10-78). The characteristics of the reversible visual disturbances are shown in table 3. The visual disturbances were often characterised by flickering light lasting less than five minutes or by general blurring of vision lasting more than 60 minutes, but they did not fall into well defined categories. The headache related to the reversible visual disturbances fulfilled the ICHD-1 for migraine without aura in 37\% (41/112), migrainous disorder without aura in 4\% (4/112), and episodic tension-type headache in $16 \%(18 / 112)$ of patients. However, $40 \%$ (45/112) of patients had unspecified headache and 3\% (4/112) had no headache. The headache related to the reversible visual disturbances began after the onset of the visual disturbances with a free interval of less than one hour in $60 \%$ (54/90), simultaneously with the visual disturbances in $14 \%$ (13/90), and before the onset of the visual disturbances in $26 \%$ (23/90) of patients (18 missing values). In $8 \%$ (9/112) of patients the reversible visual disturbances fulfilled the ICHD-1 for migrainous disorder with aura. That is, the patients fulfilled all but one of the criteria for MA.

\section{Testing of selected sets of diagnostic criteria for MA}

Initially, five sets of selected diagnostic criteria for MA were tested on 200 patients, the training sample. The diagnoses made according to the selected sets of diagnostic criteria (based on the visual aura exclusively) were compared with the diagnosis according to the ICHD-1 for MA (table 4). The set of diagnostic criteria presented at the top of table 4 was suggested by the Classification Committee of the International Headache Society. However, due to low sensitivity $(46 \%)$ this set of criteria was rejected. The set of diagnostic criteria presented at the bottom of table 4 had high sensitivity $(84 \%)$ and specificity (97\%) and was accepted by the Classification Committee for inclusion in the diagnostic criteria for MA in the ICHD-2.

\section{Validation of accepted diagnostic criteria for MA}

The accepted set of diagnostic criteria for MA was validated on 274 patients, the validation sample. The diagnosis made according to the accepted set of diagnostic criteria (based on

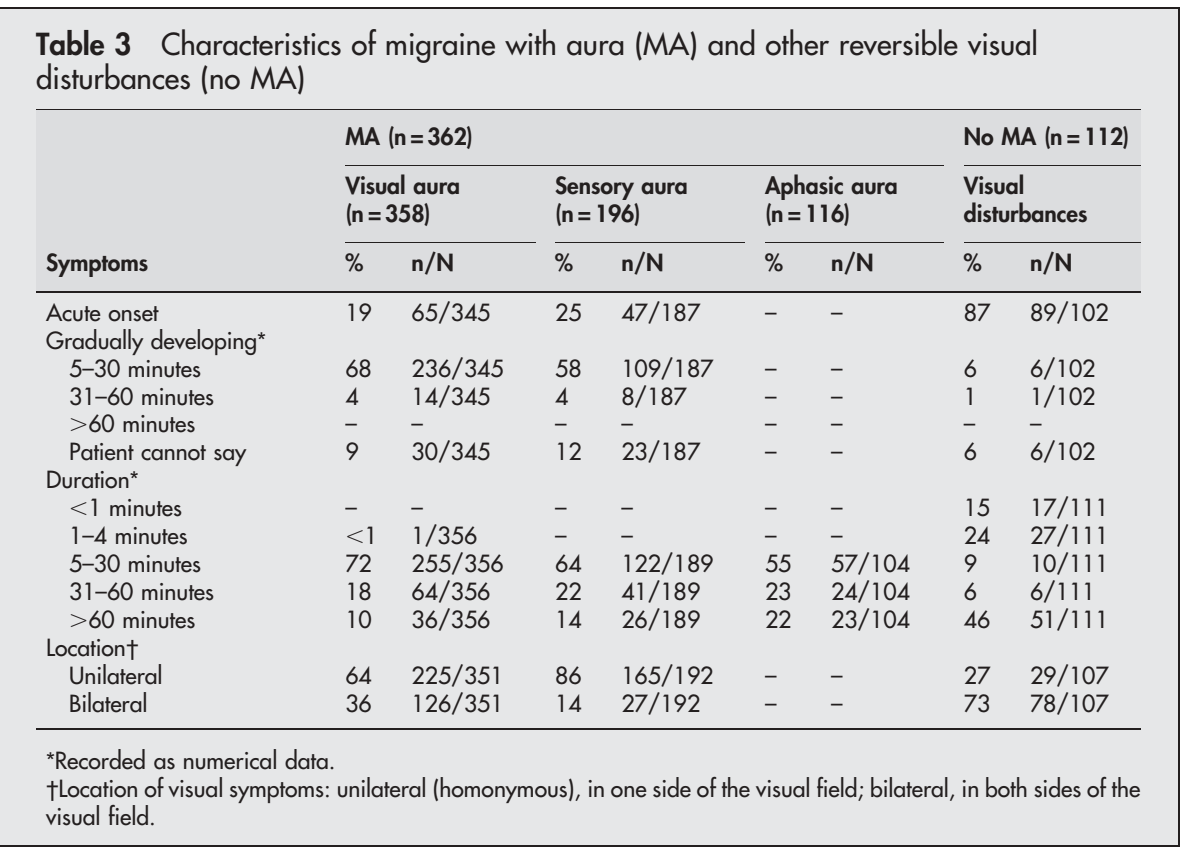


Table 4 Testing of selected sets of diagnostic criteria for migraine with aura (training sample, $n=200$ )

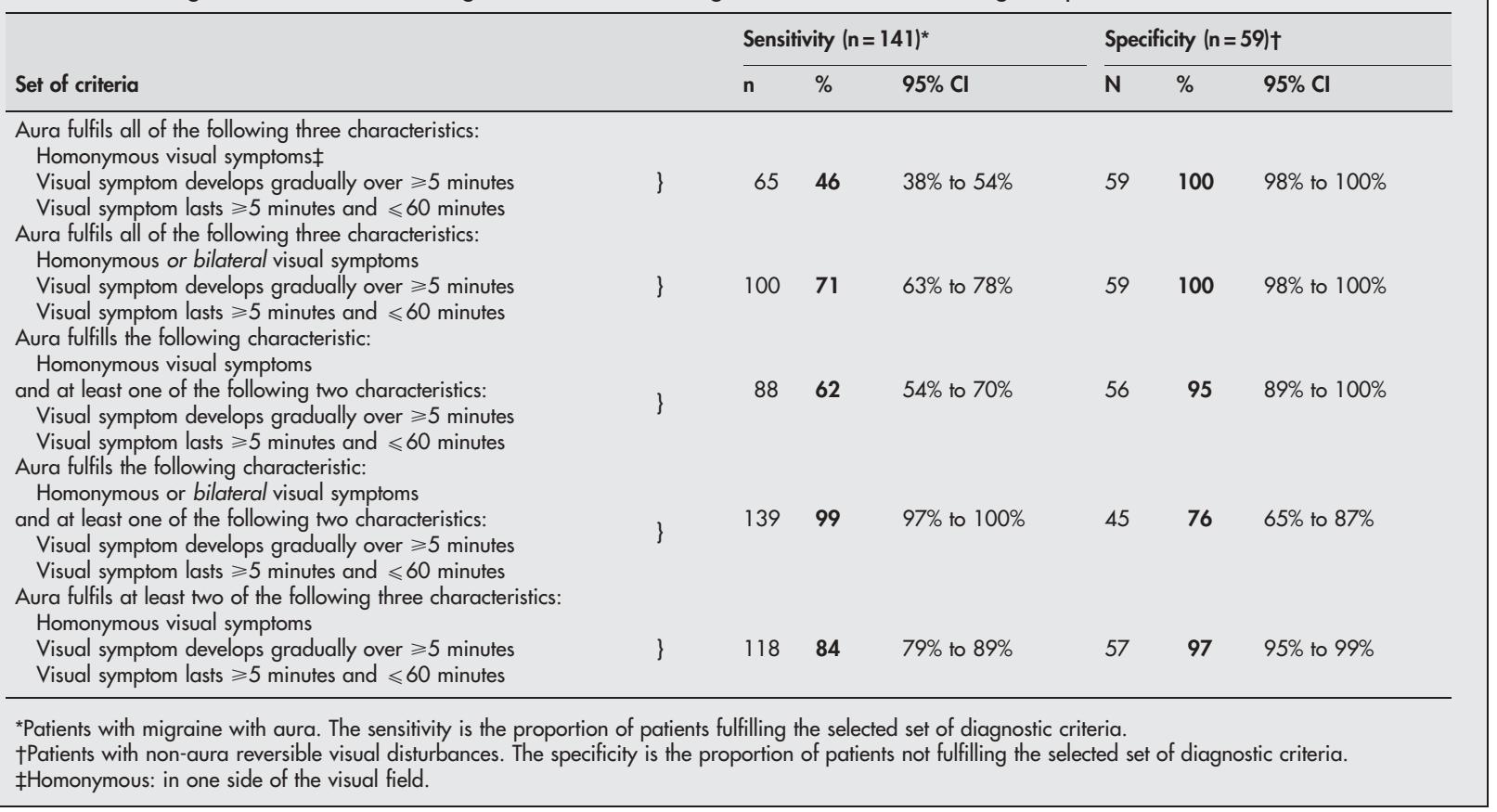

the visual, sensory, and aphasic aura) compared with the diagnosis according to the ICHD-1 for MA had a sensitivity of $90 \%$ and a specificity of $96 \%$ (table 5 ). In other words, $90 \%$ of patients with migraine aura were correctly diagnosed as having MA and $96 \%$ of patients with non-aura reversible visual disturbances were correctly diagnosed as not having MA according to the accepted set of diagnostic criteria. The likelihood ratio of a positive result of the diagnostic criteria was $23(0.90 /(1-0.96))$ and the likelihood ratio of a negative result was $0.10((1-0.90) / 0.96)$. This implies that if a patient fulfils the accepted diagnostic criteria the risk that the patient has MA is 23 times the risk that the patient does not have MA. Likelihood ratios are reliable measures as they are independent of the prevalence of MA in the study population (as opposed to predictive values).

We verified that we had chosen the set of criteria for MA with the best diagnostic accuracy among the sets of criteria tested by validating the set of criteria showing the second best diagnostic accuracy on the training sample (sensitivity $99 \%$, specificity $76 \%$ ). This set of criteria had a sensitivity of $99 \%(219 / 221)$ and a specificity of $62 \%(33 / 53)$ when applied to the validation sample.

\section{DISCUSSION}

\section{Methodological considerations}

The new diagnostic criteria for MA according to the ICHD- $2^{9}$ is based on empirical data collected for the present study.
Furthermore, we added a second sample of patients with MA and patients with non-aura visual disturbances and here present a full validation of the new diagnostic criteria.

Diagnostic criteria should be developed using one sample and tested on another to avoid random errors and false positive results. The development of the ICHD-2 for MA was based on analysis of visual symptoms only because the diagnosis of MA is most difficult in patients presenting only one aura symptom. Approximately $99 \%$ of patients with MA have visual aura at least in some attacks ${ }^{13}{ }^{15}$ and $68 \%$ of patients with MA from the general population have exclusively visual aura. ${ }^{15}$ However, the validation of the ICHD-2 for MA was based on the visual, sensory, or aphasic symptoms of aura. The ICHD-2 for MA is thus ready for application in patients with MA presenting any combination of visual, sensory, or aphasic aura.

The study population was selected from patients with MA consulting a specialist plus the affected relatives of these patients. However, the proportion of patients with MA with unilateral symptoms of aura and the duration of the symptoms of aura are identical to those in a previous population based study. ${ }^{13}{ }^{15}$ Yet, a gradual development of the aura was reported less often in the present study than in the population based study (visual aura: $81 \%$ v $97 \%$, sensory aura: $75 \%$ $v 98 \%)^{13} 15$ and more than one aura symptom was reported more often than in the population based study $(60 \% \mathrm{v}$ $31 \%) .{ }^{13}{ }^{15}$ Some of the observed variations might increase the

Table 5 Validation of accepted diagnostic criteria for migraine with aura (validation sample, $\mathrm{n}=274$ )

\begin{tabular}{|c|c|c|c|c|c|c|}
\hline \multirow[b]{2}{*}{ Set of criteria } & \multicolumn{3}{|c|}{ Sensitivity $(n=221)^{*}$} & \multicolumn{3}{|c|}{ Specificity $(n=53) \dagger$} \\
\hline & $\bar{n}$ & $\%$ & $95 \% \mathrm{Cl}$ & $\bar{n}$ & $\%$ & $95 \% \mathrm{Cl}$ \\
\hline $\begin{array}{l}\text { Aura fulfils at least two of the following three characteristics: } \\
\text { Homonymous } ¥ \text { visual symptoms and/or unilateral sensory symptoms } \\
\text { At least one aura symptom develops gradually over } \geqslant 5 \text { minutes and/or } \\
\text { different symptoms occur in succession over } \geqslant 5 \text { minutes } \\
\text { Each symptom lasts } \geqslant 5 \text { minutes and } \leqslant 60 \text { minutes }\end{array}$ & 199 & 90 & $86 \%$ to $94 \%$ & 51 & 96 & $91 \%$ to $100 \%$ \\
\hline
\end{tabular}

*Patients with migraine with aura. The sensitivity is the proportion of patients fulfilling the selected set of diagnostic criteria.

†Patients with non-aura reversible visual disturbances. The specificity is the proportion of patients not fulfilling the selected set of diagnostic criteria. †Homonymous: in one side of the visual field. 
sensitivity and some might decrease the sensitivity of the ICHD-2 for MA when applied to population samples.

The characteristics of the reversible non-aura visual disturbances were similar in patients with a related migraine headache or an unspecified headache. The visual disturbances resembled the transient visual disturbances previously reported in patients with migraine without aura. ${ }^{16}$ The prevalence of transient visual disturbances is high in both patients with migraine and healthy controls ${ }^{17}$ but the pathogenesis has not been elucidated. The positive visual symptoms-that is, flickering light in patients with migraine without aura, may be explained by a suggested lower cortical threshold for visual stimulation and presence of cortical hypersensitivity in patients with migraine. ${ }^{18} 19$

\section{Scientific implications}

The reliability of the ICHD-2 for MA is believed to be improved compared with the ICHD-1 for MA as the criteria have been further operationalised and a description of the typical symptoms of aura is included in the criteria (see table 2). As a consequence, the diagnosis of MA now relies less on clinical judgement. The classification of primary headaches according to the ICHD-1 has previously been shown to have quite good reliability but the studies included only 22 patients with MA in total. ${ }^{5620}$ Future studies will show if the ICHD-2 criteria for MA live up to the expectedly increased reliability. In the present study we aimed at identifying an equal number of patients with MA and patients with non-aura visual disturbances for the validation sample. Recruiting controls with non-aura visual symptoms from headache populations was, however, difficult and we did not get an equal number. Further testing of the MA criteria in patients with non-migraine visual or sensory disturbances would be valuable.

The validity of the ICHD-2 for MA is believed to be fair because the criteria are based on the statistical analysis of empirical data from a large sample. The diagnosis of MA in our patients was supported by a long history of MA, a history of previous diagnosis of migraine, and antimigraine treatment and a strong family predisposition to MA. ${ }^{13}{ }^{21}$ Furthermore, the criteria were developed using the cardinal characteristics of migraine aura agreed by experts and in agreement with previous empirical findings. ${ }^{15}{ }^{22-24}$ Assessment of validity is difficult when analysing the diagnostic criteria for MA because it is a clinical entity with no biological markers to confirm the diagnosis. Even the ICHD-1 for MA is not a valid gold standard as it was based on the opinion of experts and partly allowed subjective interpretations. The validation criteria must be independent from the diagnostic criteria tested. Previous validation studies of the ICHD- 1 encountered similar methodological problems..$^{25-28}$ The validity of the ICHD-1 for migraine without aura has been evaluated using logistic regression models with subjective distress as a validation criterion. ${ }^{25}{ }^{26}$ However, this criterion is generally regarded as not suitable. Eventually the validity of the criteria for MA will have to be tested against the genetic constitution of $\mathrm{MA}^{29}$ against the response to novel selective drugs such as tonabersat that might prevent cortical spreading depression, ${ }^{30}$ or against the characteristic changes in cerebral blood flow during MA attacks. ${ }^{31}{ }^{32} \mathrm{MA}$ will continue to be diagnosed on the basis of the description of symptoms until the diagnosis can be based on biological mechanisms or genetics.

The individual symptoms of aura forming the components of the ICHD-2 for MA were chosen a priori by the Classification Committee of the International Headache Society. The new criteria for MA (see table 2) are tighter than the more open criteria of the ICHD-1 (see table 1) accepting only three kinds of aura symptoms: visual, sensory, and aphasic.
Table 6 Classification of migraine according to the International Headache Society (IHS) ICHD-2 codes and the WHO ICD-10 NA codes

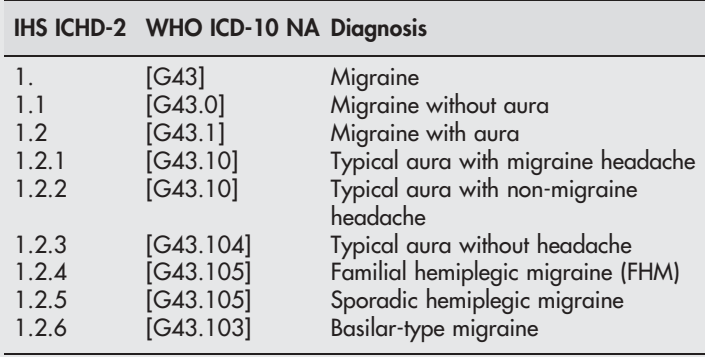

Other symptoms (except hemiplegia) additional to the typical symptoms of aura do not affect the diagnosis. Thus the new criteria will enable an analysis of how often other symptoms such as distorted vision, micropsia/macropsia, deja/jamais vue, and olfactory and auditory hallucinations may occur together with the typical symptoms of aura. It also remains to be studied how often so-called basilar-type symptoms occur together with typical symptoms of aura. An analysis of further aura characteristics using logistic regression models may reveal whether the present criteria for MA are the best option or if including other aura characteristics will further improve the validity and reliability of these criteria.

\section{Clinical implications}

The major principles of the diagnosis of MA according to the ICHD-2 have not been changed compared with the ICHD-1. This ensures continuity in the way MA is diagnosed. The existing body of evidence gained using the ICHD-1 for MA remains valid for the diagnosis made using the ICHD-2 for MA. Therefore, patients who fulfil the ICHD-2 diagnostic criteria for MA will usually respond to specific antimigraine treatment such as triptans. The ICHD-2 for MA is intended equally for research and for clinical practice and it can be used at different levels of specialisation. ${ }^{9}$ This ensures increased diagnostic reliability and promotes the research/ clinical interface.

The new criteria for MA of the ICHD-2 are more operational and probably delineate a more homogeneous sample of patients with MA than the ICHD-1. By narrowing the definition of the trait one will include only individuals likely to have similar aetiology leading to the disease phenotype. The ICHD-2 is the basis for worldwide teaching in headache classification and diagnosis and will therefore benefit patient management. ${ }^{9}$ The ICHD-2 works to destigmatise individuals with headache and to gain recognition for these disorders as neurobiological conditions. It is imperative for the success of these efforts that researchers and clinicians use the same diagnostic system and that this system is as precise as possible.'

The International Headache Society was involved in developing the ICD-10 for neurological disorders (ICD-10 NA). ${ }^{73}$ The ICD-10 NA is not meant to compete with the ICHD-2 classification, but is intended to allow users to transfer their data from one system to the other to take advantage of the universally accepted coding system of the ICD-10 NA and the more detailed subdivisions of the ICHD-2 classification' (table 6).

Alhough the ICHD-2 for MA describes the common features of MA it still takes specialised neurological knowledge to diagnose the more challenging presentations of MA. In the ICHD-2 for MA the presence or absence of a headache and the nature of the headache is used only for subdiagnosing in patients with MA (see table 2). A headache is 
not essential to establish the MA diagnosis, though a headache following the aura strengthens the diagnosis of migraine. Diagnostic caution is required when aura is not followed by headache and in patients with sensory and aphasic symptoms without visual aura. ${ }^{13}$ In such cases and in cases with other diagnostic uncertainty, appropriate investigations should be undertaken to rule out intracranial pathology even if the patient fulfils the ICHD-2 criteria for MA.

\section{ACKNOWLEDGEMENTS}

The authors thank the participants, medical students I Andersen, F Nazim, and S Roemer, and many other colleagues for their excellent collaboration.

\section{Authors' affiliations}

M K Eriksen, L L Thomsen, J Olesen, Danish Headache Center, University of Copenhagen, Department of Neurology, Glostrup Hospital, Copenhagen, Denmark

This study was supported by grants from the University of Copenhagen, the Lundbeck Foundation, the Jeppe Juhl Foundation, the AP Møller Foundation for advancement of medical science, the Foundation for Research in Neurology, the Codan Foundation, the International Headache Society, and the GSK Foundation for headache research.

Competing interests: none declared

\section{REFERENCES}

1 Headache Classification Committee of the International Headache Society. Classification and diagnostic criteria for headache disorders, cranial neuralgias and facial pain. Cephalalgia 1988;8:1-96.

2 Edmeads J, Findlay $\mathrm{H}$, Tugwell $\mathrm{P}$, et al. Impact of migraine and tension-type headache on life-style, consulting behaviour, and medication use: a Canadian population survey. Can J Neurol Sci 1993;20:131-7.

3 Henry P, Michel P, Brochet B, et al. A nationwide survey of migraine in France: prevalence and clinical features in adults. GRIM. Cephalalgia. 1992;12: 22937; discussion 186

4 Treatment of migraine attacks with sumatriptan. The Subcutaneous Sumatriptan International Study Group. N Engl J Med 1991;325:316-21.

5 Russell MB, Rasmussen BK, Brennum J, et al. Presentation of a new instrument the diagnostic headache diary. Cephalalgia 1992;12:369-74.

6 Leone M, Filippini G, D'Amico D, et al. Assessment of International Headache Society diagnostic criteria: a reliability study. Cephalalgia 1994;14:280-4.

7 World Health Organization. Application of the International Classification of Diseases to Neurology, 2nd edn., Genova: World Health Organization 1997.

8 Olesen J. Classification of Headaches. In: Olesen J, Tfelt-Hansen P, Welch K, eds. The Headaches, 2nd edn. Philadelphia: Lippincot Williams \& Wilkins, 2000:13-14

9 International Classification of Headache Disorders, 2nd edn. Cephalalgia 2004; $24: 1-160$.

10 Thomsen LL, Eriksen MK, Roemer SF, et al. A population-based study of familial hemiplegic migraine suggests revised diagnostic criteria. Brain 2002; 125(Pt 6):1379-91.
11 Thomsen LL, Ostergaard E, Olesen J, et al. Evidence for a separate type of migraine with aura: sporadic hemiplegic migraine. Neurology 2003;60:595-601.

12 Lykke Thomsen L, Kirchmann Eriksen M, Faerch Romer S, et al. An epidemiological survey of hemiplegic migraine. Cephalalgia 2002;22:361-75

13 Eriksen M, Thomsen L, Olesen J. Clinical characteristics of 362 patients with familial migraine with aura. Cephalalgia 2004; 24:564-75.

14 Russell MB, Rasmussen BK, Thorvaldsen $\mathrm{P}$, et al. Prevalence and sex-ratio of the subtypes of migraine. Int J Epidemiol 1995;24:612-18.

15 Russell MB, Olesen J. A nosographic analysis of the migraine aura in a general population. Brain 1996;1 19(Pt 2):355-61.

16 Cologno D, Torelli P, Manzoni GC. Transient visual disturbances during migraine without aura attacks. Headache 2002;42:930-3.

17 Mattsson P, Lundberg PO. Characteristics and prevalence of transient visual disturbances indicative of migraine visual aura. Cephalalgia 1999:19:479-84.

18 Mulleners WM, Chronicle EP, Palmer JE, et al. Suppression of perception in migraine: evidence for reduced inhibition in the visual cortex. Neurology 2001;56:178-83

19 Mulleners WM, Aurora SK, Chronicle EP, et al. Self-reported photophobic symptoms in migraineurs and controls are reliable and predict diagnostic category accurately. Headache 2001;41:31-9.

20 Granella F, D'Alessandro R, Manzoni GC, et al. International Headache Society classification: interobserver reliability in the diagnosis of primary headaches. Cephalalgia 1994;14:16-20.

21 Kendell RE. Clinical validity. Psychol Med 1989;19:45-55.

22 Russell MB, Rasmussen BK, Fenger K, et al. Migraine without aura and migraine with aura are distinct clinical entities: a study of four hundred and eighty-four male and female migraineurs from the general population. Cephalalgia 1996;16:239-45.

23 Jensen K, Tfelt-Hansen P, Lauritzen $M$, et al. Classic migraine. A prospective recording of symptoms. Acta Neurol Scand 1986;73:359-62.

24 Manzoni GC, Farina S, Lanfranchi $M$, et al. Classic migraine-clinical findings in 164 patients. Eur Neurol 1985;24:163-9.

25 Merikangas KR, Dartigues JF, Whitaker A, et al. Diagnostic criteria for migraine. A validity study. Neurology 1994:44(6 suppl 4):S1 1-16.

26 Merikangas KR, Whitaker AE, Angst J. Validation of diagnostic criteria for migraine in the Zurich longitudinal cohort study. Cephalalgia 1993;13(suppl 12): $47-53$

27 Michel P, Dartigues JF, Henry P, et al. Validity of the International Headache Society criteria for migraine. GRIM. Groupe de Recherche Interdisciplinaire sur la Migraine. Neuroepidemiology 1993;12:51-7.

28 Iversen HK, Langemark M, Andersson PG, et al. Clinical characteristics of migraine and episodic tension-type headache in relation to old and new diagnostic criteria. Headache 1990;30:514-19.

29 Wessman M, Kallela M, Kaunisto MA, et al. A susceptibility locus for migraine with aura, on chromosome 4q24. Am J Hum Genet 2002;70:652-62.

30 Bradley DP, Smith MI, Netsiri C, et al. Diffusion-weighted MRI used to detect in vivo modulation of cortical spreading depression: comparison of sumatriptan and tonabersat. Exp Neurol 2001;172:342-53.

31 Olesen J, Larsen B, Lauritzen M. Focal hyperemia followed by spreading oligemia and impaired activation of $\mathrm{rCBF}$ in classic migraine. Ann Neurol 1981;9:344-52.

32 Cutrer FM, Sorensen AG, Weisskoff RM, et al. Perfusion-weighted imaging defects during spontaneous migrainous aura. Ann Neurol 1998;43:25-31.

33 Orgogozo J, Van Drimmelen-Krabbe J, Bradley W, et al. The World Health Organization view on disease classification and diagnosis. In: Olesen J, eds. Headache Classification and Epidemiology. New York: Raven Press, Ltd, 1994:27-35. 